\title{
Surface plasmon-like modes on structured perfectly conducting surfaces
}

\author{
Yung-Chiang Lan \\ Institute of Electro-Optical Science and Engineering, National Cheng Kung University, Tainan 701, Taiwan, \\ Republic of China \\ lanyc@mail.ncku.edu.tw \\ Ruey-Lin Chern \\ Institute of Applied Mechanics, National Taiwan University, Taipei 106, Taiwan, Republic of China
}

\begin{abstract}
Surface plasmon-like (SPL) modes are the electromagnetic surface eigenmodes supported by the structured perfectly conducting surfaces. The standard eigenvalue-solving method is adopted to solve these SPL modes. The field patterns of the SPL modes in the square holes for inplane wavevectors $k_{x}=2 \pi / 2 d$ and $k_{x}=2 \pi / d$ are $\mathrm{TE}_{10}$-like and $\mathrm{TE}_{11}$, respectively. However, the field patterns can no longer be identified as any particular waveguide mode for other in-plane wavevectors. The dispersion relations of the SPL modes are obtained numerically. The change in mode character with wavevector prevents the dispersion relation from being derived by assuming only the fundamental mode in the holes. On a thin perfect conductor perforated with structures, the SPL mode splits into a high-frequency anti-symmetric mode and a low-frequency symmetric mode, which is caused by the mutual interaction of the electromagnetic evanescent fields on both sides.
\end{abstract}

(C)2006 Optical Society of America

OCIS codes: (240.6680) Surface plasmons; (240.6690) Surface waves

\section{Reference and links}

1. H. Raether, Surface Plasmons on Smooth and Rough Surfaces and on Gratings (Springer, Berlin, 1988).

2. D. Sarid, "Long-range surface-plasma waves on very thin metal films," Phys. Rev. Lett. 47, 1927-1930 (1981).

3. W. L. Barnes, A. Dereuux, and T. W. Ebbesen, "Surface plasmon subwavelength optics," Nature 424, 824830 (2003).

4. E. Ozbay, "Plasmonics: merging photonics and electronics at nanoscale dimensions," Science 311, 189-193 (2006).

5. A. P. Hibbins, J. R. Sambles, and C. R. Lawrence, "Grating-coupled surface plasmons at microwave frequencies," J. Appl. Phys. 86, 1791-1795 (1999).

6. F. Miyamaru and M. Hangyo, "Strong enhancement of terahertz transmission for a three-layer heterostructure of metal hole arrays," Phys. Rev. B 72, 035429 (2005).

7. J. G. Rivas, C. Schotsch, P. H. Bolivar, and H. Kurz, "Enhanced transmission of THz radiation through subwavelength holes," Phys. Rev. B 68, 201306 (2003).

8. H. Cao and A. Nahata, "Resonantly enhanced transmission of terahertz radiation through a periodic array of subwavelength apertures," Opt. Express 12, 1004-1010 (2004).

9. J. B. Pendry, L. Martin-Moreno, and F. J. Garcia-Vidal, "Mimicking surface plasmons with structured surfaces," Science 305, 847-848 (2004).

10. F. J. Garcia-Vidal, L. Martin-Moreno, and J. B. Pendry, "Surfaces with holes in them: new plasmonic metamaterials," J. Opt. A: Pure Appl. Opt 7, S97-S101 (2005).

11. W. Barnes and R. Sambles, "Only skin deep," Science 305, 785-786 (2004).

12. A. P. Hibbins, B. R. Evans, and J. R. Sambles, "Experimental verification of designer surface plasmons," Science 308, 670-672 (2005).

13. A. P. Hibbins, M. J. Lockyear, I. R. Hooper, and J. R. Sambles, "Waveguide arrays as plasmonic metamaterials: transmission below cutoff," Phys. Rev. Lett. 96, 073904 (2006). 
14. F. J. García de Abajo and J. J. Sáenz, "Electromagnetic surface modes in structured perfect-conductor surfaces,” Phys. Rev. Lett. 95, 233901 (2005).

15. W. H. Press, B. P. Flannery, S. A. Teukolsky, and W. T. Vetterling, Numerical Recipes in FORTRAN: The Art of Scientific Computing (Cambridge University Press, New York, 1992).

16. A. Taflove and S. C. Hagness, Computational Electrodynamics: The Finite-Difference Time-Domain Method $2^{\text {nd }}$ Ed. (Artech House, Norwood, 2000).

17. Y. C. Lan, "Optical tunneling effect of localized surface plasmon: a simulation study using particle-in-cell method," Appl. Phys. Lett. 88, 071109 (2006).

18. C. K. Birdsall and A. B. Langdon, Plasma Physics via Computer Simulation (Institute of Physics Publishing, London, 1991).

19. C. S. Lee, S. W. Lee, and S. L. Chuang, "Plot of modal field distribution in rectangular and circular waveguides," IEEE Trans. Microwave Theory Tech. 33, 271-274 (1985).

20. K. Sakoda, Optical Properties of Photonic Crystals (Springer, Berlin, 2001).

21. F. J. García de Abajo, J. J. Sáenz, I. Campillo, and J. S. Dolado, "Site and lattice resonances in metallic hole arrays," Opt. Express 14, 7-18 (2006).

22. L. Martin-Moreno, F. J. Garcia-Vidal, H. J. Lezec, K. M. Pellerin, T. Thio, J. B. Pendry, and T. W. Ebbesen, "Theory of extraordinary optical transmission through subwavelength hole arrays," Phys. Rev. Lett 86, 1114-1117 (2001)

\section{Introduction}

Surface plasmon (SP) is the coherent fluctuations of the electron charges at the metal-air (or dielectric) interface [1]. These charge fluctuations represent electromagnetic surface modes with the fields highest at the interface and decaying exponentially into both media. On a flat metal surface, the SP cannot be directly excited by incident radiation, because the dispersion curve of the SP lies to the right of the light line. A periodic metallic structure can, however, provide the additional in-plane momentum required by the incident light in an appropriate polarization state to excite the SP, causing strong optical absorption [1]. Surface plasmons are also present on the thin metal films. However, since the electromagnetic fields on both surfaces interact with each other, the SP splits into a high-frequency anti-symmetric mode and a low-frequency symmetric mode [1,2]. Given these interesting and useful interactions between the light and the SP, plasmonics have drawn much attention because of their potential application in nano-scale optics [3,4].

SP can not exist on perfectly conducting planar surfaces because the incident field is screened out completely by the perfect conductor. However, the surface plasmon-like (SPL) behavior has also been found in almost plasmon-free good conductor films with periodic holes' array at microwave and $\mathrm{THz}$ frequencies [5-8]. In these experiments, the surface plasmon-like behavior is provided mainly by the periodic structure instead of the metal's real surface plasmon because the incident wave frequency is far below the plasma frequency of the metal. Recently, Pendry et al. proposed the formation of perfect conductor support surface modes by drilling an array of holes in the surface, associated with the behavior of the SP [911]. Subsequently, Hibbins et al. experimentally confirmed this SPL mode at the microwave region $[12,13]$. In Pendry's scheme, only the $\mathrm{TE}_{10}$ waveguide mode is assumed to be present in the square holes because it is the fundamental mode with the most weakly decaying behavior $[9,10]$. The frequency of the incident wave for simulating the exponential decay of the electromagnetic field in the metal must be below the cutoff frequency of the $\mathrm{TE}_{10}$ mode. After the electromagnetic fields are matched across the surface, the canonical plasmon frequency and dispersion relation of the SPL mode are derived. García de Abajo et al. applied the plane wave expansion approach to yield the surface-bound modes by matching the electromagnetic fields in terms of the diffracted plane waves outside the surface and the waveguide modes inside the holes [14]. They demonstrated that these surface-bound modes cannot be generally represented by local, frequency-dependent optical constants. However, in the literature, the field patterns of the SPL modes on the structured conductor surfaces, the size effect of the holes on the SPL modes and the SPL modes on a thin structured perfect conductor have rarely been discussed. (Like the SP on a thin metal film, it is also possible for SPL mode to split into two modes on a thin structured perfect conductor.) 
In this work, a new perspective is taken to study the SPL modes on a structured perfectly conducting surface. Like the SP on a metal surface, the SPL modes are supposed to be the electromagnetic surface eigenmodes supported by the structured conductors. Therefore, the standard eigenvalue-solving approach can be used to solve Maxwell's equations with suitable boundary conditions [15].

This paper is organized as follows. The simulation model and method are described in Section 2. In Section 3, the simulation results and discussions are presented. Finally, the conclusions are given in Section 4.

\section{Simulation methods}

The finite-difference time-domain (FDTD) method with a pulsed driving source is first adopted to obtain the spectrum of the eigenmodes [16]. Then, the eigenmode solver based on the same FDTD grids is employed to elucidate the electromagnetic field patterns. An electron beam above the conductor surface is introduced to excite these modes, similar to that in the SP, to confirm that these SPL modes are the real surface eigenmodes. The particle-in-cell (PIC) method is utilized to simulate the electron beam's passing through the surface and the interaction between the electron beam and the SPL modes [17, 18]. The PIC method uses a finite-size macroparticle to represent a group of real electrons. The motion of the macroparticles is explicated by integrating the equations of motion. The FDTD scheme based on the "Yee grid" [16] is used to determine self-consistently the interaction between the electromagnetic fields and the plasma particles.

Figure 1 presents the simulated structure of repeated units of a square perfect conductor with a square hole. The period of the unit $d$ is $10 \mathrm{~mm}$. The width of the square holes $w$ is changed from $1 \mathrm{~mm}$ to $9 \mathrm{~mm}$ to explore the effect of the hole-size on the field patterns and the dispersion relations of the SPL modes. The depth of the holes $t$ is $30 \mathrm{~mm}$, which is deep enough to avoid any significant change in the field patterns and frequencies of the SPL modes. The three-dimensional Cartesian coordinate system is used in the simulation. The boundaries in the $\mathrm{x}$ - and $\mathrm{y}$-directions are set as the periodic boundaries to represent the infinite repeated units. The boundaries in the z-direction are the wave absorption boundaries.

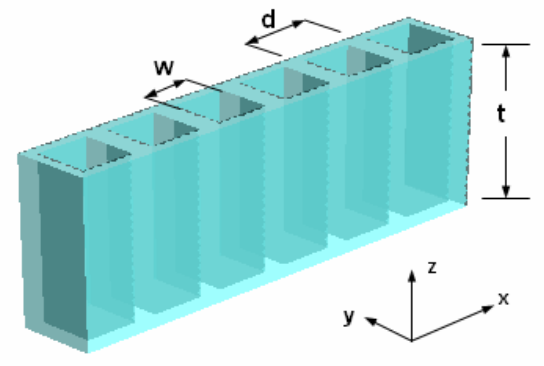

Fig. 1. Schematic simulated structure. The period of the repeated units $d$ and the depth of the square holes $t$ are $10 \mathrm{~mm}$ and $30 \mathrm{~mm}$, respectively. The width of the holes $w$ is changed from $1 \mathrm{~mm}$ to $9 \mathrm{~mm}$.

\section{Results and discussions}

The space variations of the electromagnetic fields of the SPL modes on the holey perfectconductor surface are initially studied. Figures 2(a) and 2(b) plot the electric field vectors on the conductor surface for in-plane (x-component) wavevectors $k_{x}=2 \pi / 2 d$ and $k_{x}=2 \pi / d$, respectively, with a hole width of $7 \mathrm{~mm}$. The in-plane wavevectors $k_{x}=2 \pi / 2 d$ 

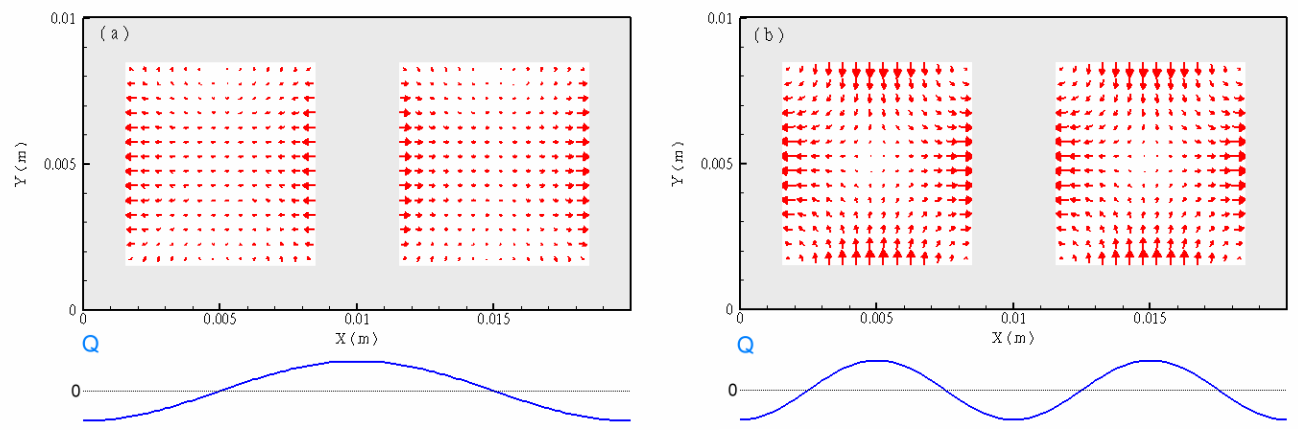

Fig. 2. Electric field vectors of SPL modes on holey perfect-conductor surfaces and variations of equivalent charges Q with x-position: (a) $k_{x}=2 \pi / 2 d$ and (b) $k_{x}=2 \pi / d$, for the holes of width $7 \mathrm{~mm}$ and depth $30 \mathrm{~mm}$.

and $k_{x}=2 \pi / d$ mean the wavelengths of the SPL mode are $2 d$ and $d$, respectively. Figures 2 also present the variations of the equivalent charges $\mathrm{Q}$ with the $\mathrm{x}$-position. Figure 2 clearly demonstrates that the dominant field pattern in the square hole for $k_{x}=2 \pi / 2 d$ is a $\mathrm{TE}_{10}$-like mode (although it is not really a $\mathrm{TE}_{10}$ mode pattern) [9, 19]. However, for $k_{x}=2 \pi / d$, the dominant field pattern in the square hole is the $\mathrm{TE}_{11}$ mode rather than the $\mathrm{TE}_{10}$ mode [19]. Even in a much smaller hole, such as one of width $1 \mathrm{~mm}$, the field patterns (which are not displayed here) remain like those in the hole with a width of $7 \mathrm{~mm}$. The $k_{x}=2 \pi / d$ mode has the $\mathrm{TE}_{11}$ pattern because the translation symmetry in the $\mathrm{x}$-direction and the electric field must be normal to the perfectly conducting surface, requiring the directions of the electric fields on the left and right sides of the hole to be opposed (as shown in Fig. 2(b)). Accordingly, only the $\mathrm{TE}_{11}$ modes are allowable in the hole. This field pattern can be viewed as an effective electric quadrupole on the perfectly conducting surface, consisting of one pair of positive charges on one pair of opposite sides of the hole and another pair of negative charges on the other pair of opposite sides of the hole. If the $\mathrm{TE}_{10}$ mode is present in the hole, then the symmetry will require the opposite equivalent charges to appear simultaneously on the conductor surface between the two adjacent holes. This result is unreasonable so the $\mathrm{TE}_{10}$ mode is not allowable. In the case of $k_{x}=2 \pi / 2 d$, as shown in Fig. 2(a), the electromagnetic fields of the surface modes on the two neighboring holes will interact with each other, causing the field pattern to be similar to that of the $\mathrm{TE}_{10}$ mode, and with equivalent opposite charges alternately distributed on the conductor surface. For other values of $k_{x}$, the field coupling between neighboring holes becomes very complicated and the field patterns can no longer be identified as a particular mode pattern. Figure 3 plots the amplitudes of the $E_{x}$ field (x component of the electric field) against $\mathrm{z}$ position (along the line through the center of the square hole), for $k_{x}=2 \pi / 2 d$ and $k_{x}=2 \pi / d$, with a hole width of $7 \mathrm{~mm}$. In Fig. 3, the curves of $E_{x}$ against $\mathrm{z}$ position are also fitted into the exponential functions. Figure 3 indicates that the fields of the SPL modes are highest at the surface of the structured conductor $(\mathrm{z}=0)$ and decay exponentially into both the air and the hole with different decay constants. These results confirm the characteristics of the surface eigenmodes, which are very like the SP excited at the metal-air interface.

Next, the dispersion relations of the SPL modes are investigated. Figure 4 plots the dispersion relations for three widths of holes $-5 \mathrm{~mm}, 7 \mathrm{~mm}$ and $9 \mathrm{~mm}$. Figure 4 also presents the light line for comparison. As shown in Fig. 4, the dispersion relations of the SPL modes asymptotically approach the light line from the right at low frequencies (like the SP). Figure 4 also exhibits that the dispersion relations of the SPL mode approach the light line as the width 


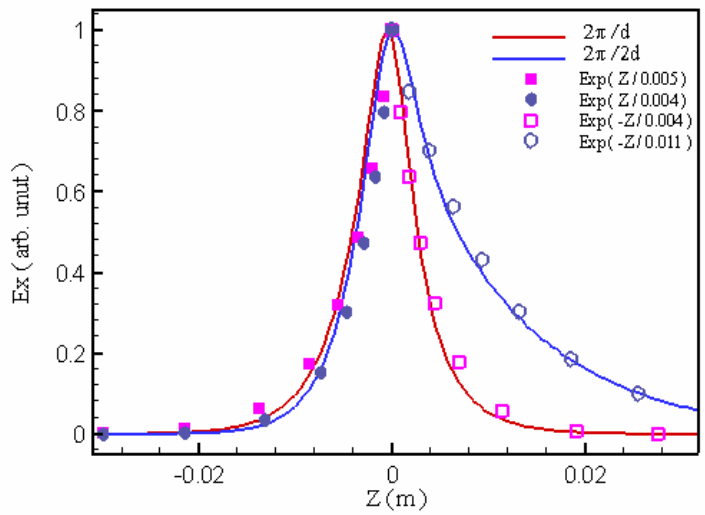

Fig. 3. Amplitudes of $E_{x}$ field against z position for SPL modes with the holes of width $7 \mathrm{~mm}$, a depth of $30 \mathrm{~mm}$ and in-plane wavevectors of $k_{x}=2 \pi / 2 d$ and $k_{x}=2 \pi / d$. The decays of the field on both sides of the peak are fitted to the exponential functions.

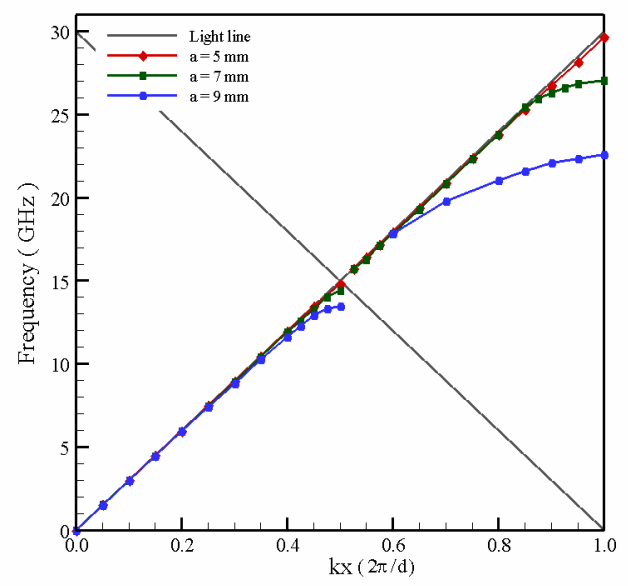

Fig. 4. Dispersion relations of SPL modes on holey perfect-conductor surfaces with holes of three widths $-5 \mathrm{~mm}, 7 \mathrm{~mm}$ and $9 \mathrm{~mm}$. The light line is also presented for comparison.

of the holes decreases from $9 \mathrm{~mm}$ to $5 \mathrm{~mm}$. As the wavelength increases (the frequency and $k_{x}$ decline) or the hole width decreases, the ratio of the hole width to the wavelength decreases (such that the hole can be regarded as a small perturbation to the wave on the surface), and the surface mode is therefore more like the light. Furthermore, the dispersion relations divide into two branches is observed in Fig. 4. In the first branch, the in-plane wavevector $k_{x}$ ranges from 0 to $2 \pi / 2 d$ and the dispersion relations are bent when $k_{x}$ approximates to $2 \pi / 2 d$. The frequency depression close to $k_{x}=2 \pi / 2 d$ is typical of a onedimensional photonic crystal with the in-plane periodicity, which results from the mixing of modes with $k_{x} \approx 2 \pi / 2 d$ and $k_{x} \approx-2 \pi / 2 d$ in the presence of the periodic boundaries (i.e. the Bragg diffraction at the first Brillouin zone boundary) [20]. On the other hand, the inplane wavevector $k_{x}$ in the second branch ranges from $2 \pi / 2 d$ to $2 \pi / d$. However, except 
for $k_{x}=2 \pi / d$ or smaller hole widths (such as $5 \mathrm{~mm}$ ), the quality factors of the second branch of the SPL modes are very small and those modes are very difficult to be excited by the electron beams. And except for $k_{x}=2 \pi / d$, the $E_{x}$ fields of the surface modes decay exponentially into the holes but oscillate in the air. These phenomena come from the fact that this branch of the surface modes is coupled to the radiation modes, which have been pointed out by Barnes et al. in Ref. [11]. Therefore, the surface modes in the second branch are not truly the SPL modes. Only the $k_{x}=2 \pi / d$ mode is the real SPL mode with the $\mathrm{TE}_{11}$ mode pattern. In fact, the dispersion relations obtained here are very similar to those in García de Abajo's work [14, 21]. And the second branch in Fig. 4 is correspondent with their first diffraction order branch in Ref. [21]. The dispersion relations of the SPL modes obtained in this study are also compared with Pendry's analytical equation derived in Ref. [9]. Figure 5 plots the first branch of the dispersion relations in Fig. 4 and the analytical dispersion relations in Ref. [9] for three widths of holes $-5 \mathrm{~mm}, 7 \mathrm{~mm}$ and $9 \mathrm{~mm}$. (Here only the first branch is drawn because the field pattern in the hole is the $\mathrm{TE}_{10}$-like mode for $k_{x}=2 \pi / 2 d$.) Figure 5 shows that the dispersion relations obtained here agree with Pendry's predictions well at smaller frequencies (longer wavelength) and smaller holes' sizes. And this result is expected because the long-wavelength limit $\left(a<d<<\lambda_{0}\right.$ ) is assumed when the analytical dispersion relation is derived $[9,10]$. Besides, the interhole interaction, discussed above, causes the field pattern in the hole to change with $k_{x}$ and not to belong generally to any standard waveguide mode. Hence, the dispersion relation of the SPL mode on the holey perfect conductor cannot be generally derived by assuming only the fundamental mode in the holes $[9,14]$.

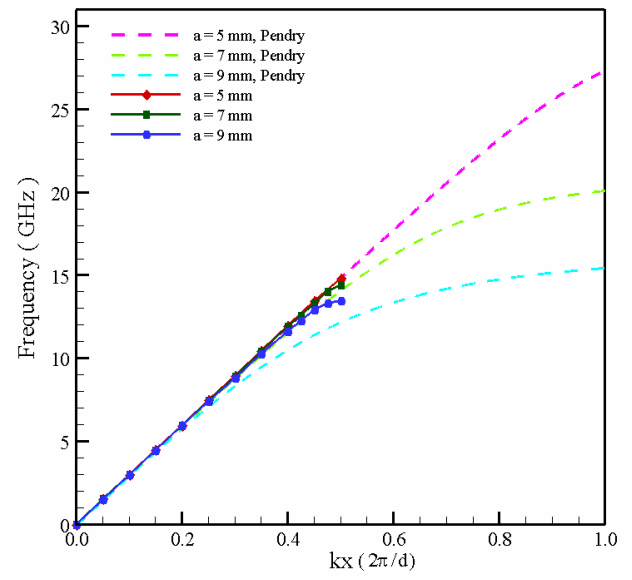

Fig. 5. First branch of dispersion relations of SPL modes in Fig. 4. The analytical dispersion relations derived in Ref. [9] are also presented by dashed lines for comparison.

Finally, the characteristics of the SPL modes on a thin structured perfect conductor are examined. The simulated structure is presented in Fig. 6. The perfect conductor is $4 \mathrm{~mm}$ thick and perforated with square holes of width $7 \mathrm{~mm}$ (the period is still $10 \mathrm{~mm}$ ). Figure 7 displays the amplitudes of the $E_{x}$ field as a function of the $\mathrm{z}$ position for two surface eigenmodes with the same in-plane wavevector $k_{x}=2 \pi / 2 d$ (the origin of the z-axis at the mid depth in the hole). As presented in Fig. 7, the SPL mode on the thin conductor splits into 


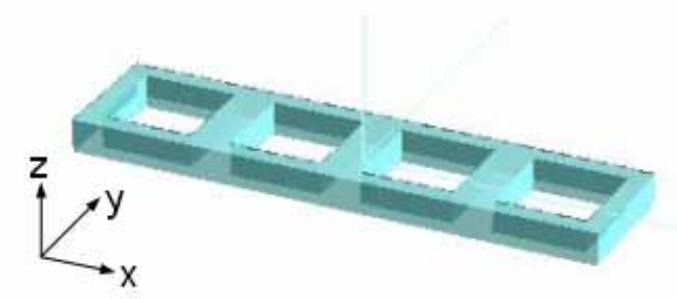

Fig. 6. Schematic simulated structure for SPL modes on a thin structured perfect conductor. The period of the repeated units, the width of the hole and the thickness of the conductor are $10 \mathrm{~mm}, 7 \mathrm{~mm}$ and $4 \mathrm{~mm}$, respectively.

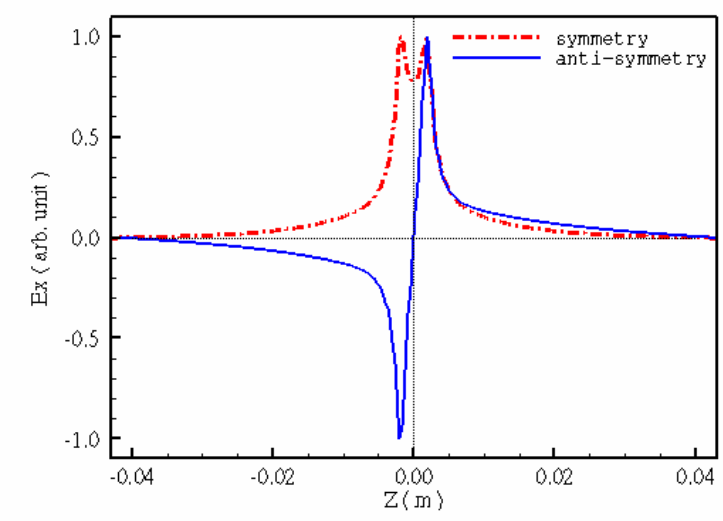

Fig. 7. Amplitudes of $E_{x}$ field as a function of z position for SPL modes on a $4 \mathrm{~mm}$ thick, holey perfect conductor with holes of width $7 \mathrm{~mm}$ and in-plane wavevector $k_{x}=2 \pi / 2 d$. The origin of the $\mathrm{z}$-axis is located at the mid depth in the hole.

one symmetrical mode ( $L^{-}$mode, with the field symmetrical to $\mathrm{z}=0$ ) and one antisymmetrical mode ( $L^{+}$mode, with the field anti-symmetrical to $\mathrm{z}=0$ ). The frequencies of the $L^{-}$mode, the $L^{+}$mode and the SPL mode on the $30 \mathrm{~mm}$-thick perfect conductor, $\omega^{-}$, $\omega^{+}$and $\omega$, respectively, follow the order $\omega^{-}<\omega<\omega^{+}$, which corresponds to the SP on a thin metal surface [2]. Also like that of the SP, the mode splitting is caused by the interaction of electromagnetic evanescent fields on both surfaces [22]. Figure 8 plots the dispersion relations of the symmetrical and anti-symmetrical SPL modes on a thin perfect conductor with the geometry and dimensions shown in Fig. 6. For comparison, the light line and dispersion relations of the SPL modes on the $30 \mathrm{~mm}$-thick holey perfect conductor are also plotted in

Fig. 8. Figure 8 displays clearly the frequency order $\omega^{-}<\omega<\omega^{+}$discussed above in each branch of the dispersion relations. And the mode splitting becomes more obvious as the inplane wavevector $k_{x}$ increases for both the first and second branches of the dispersion relations. These frequency relations are also very similar to those of the SP on a thin metal film. As stated above, the SPL modes should be excited by an electron beam. For verifying the SPL mode splitting on a thin structured perfect conductor further, a numerical experiment that introduce an electron sheet into a simulation structure with only two repeated units is carried out. The electron sheet that moves in the $\mathrm{x}$-direction is launched from the lower $\mathrm{x}$ boundary and absorbed by the upper $x$ boundary. The electron sheet is located $2 \mathrm{~mm}$ above the conductor surface with a width of $4 \mathrm{~mm}$ and an incident energy and current of $10 \mathrm{eV}$ and 1 


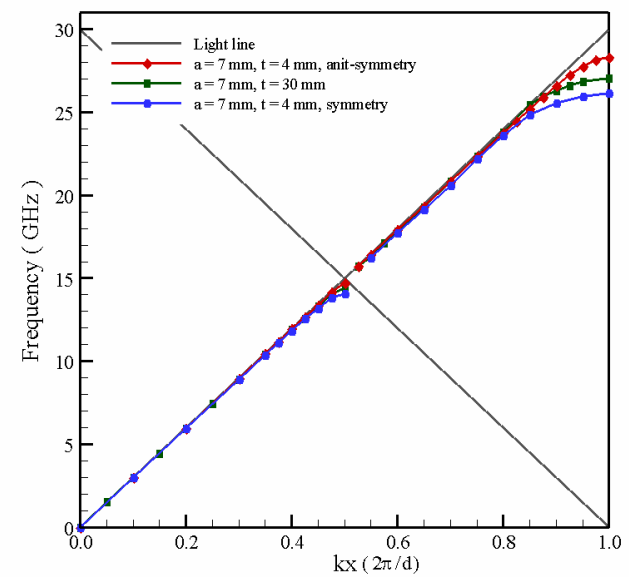

Fig. 8. Dispersion relations of SPL modes on a thin perfect conductor perforated with square holes. The structure and dimensions are given in Fig. 6. The light line and the dispersion relation of the SPL modes on holey perfect-conductor surfaces with the holes of width $7 \mathrm{~mm}$ and depth $30 \mathrm{~mm}$ shown in Fig. 4 are also presented for comparison.

A, respectively. The simulation structure (with the same dimensions as in Fig. 6) and the snapshot of the electron sheet are presented in Fig. 9. (As in Fig. 1, the boundaries in the Xand y-directions are also set as the periodic boundaries.) Figure 10 plots the spectrum of the $E_{x}$ field measured at the center of the hole near the lower interface. Figure 10 displays clearly that the SPL mode splits into two modes on a thin structured perfect conductor for both in-plane wavevectors $k_{x}=2 \pi / 2 d$ and $k_{x}=2 \pi / d$. The electron sheet provides sufficient in-plane momentum and energy to excite the SPL modes, but the symmetry of the two repeated units is such that only the SPL modes with $k_{x}=2 \pi / 2 d$ and $2 \pi / d$ are supported.

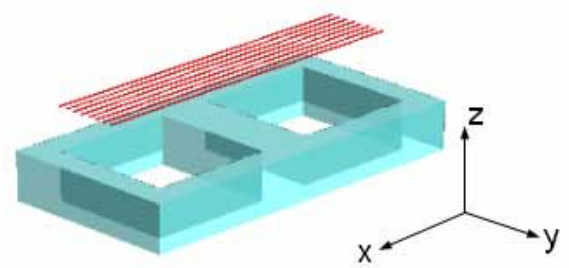

Fig. 9. Simulation structure and snapshot of electron sheet for the numerical experiment of SPL modes excited by the electron sheet. The dimensions of the structure are given in Fig. 6.

If the conductor has a finite conductivity or the hole is filled with a dielectric (which cases are not discussed here), then the frequencies of the SPL modes will shift, but the proposed scheme is still applicable. The SPL mode also exists on a real metal (such as silver) with nano-scale holes at visible light frequencies. For example, if the period of the unit and the width of the square hole are reduced to $100 \mathrm{~nm}$ and $70 \mathrm{~nm}$, respectively, the frequency of the SPL mode corresponding to $k_{x}=2 \pi / 2 d$ increases to $14.4 \times 10^{14} \mathrm{~Hz}$. In such a case, distinguishing the SP from the SPL mode becomes difficult since their effects are coupled 


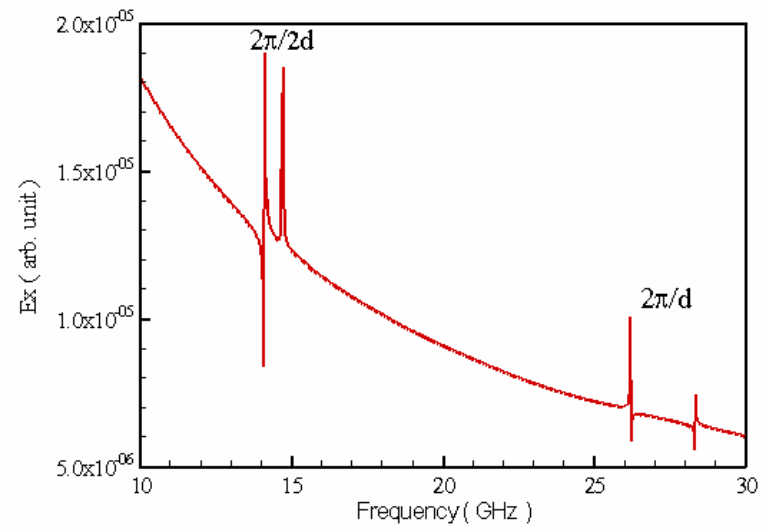

Fig. 10. Spectrum of the $E_{x}$ field measured at the center of the hole near the lower interface for the SPL modes excited by the electron sheet. The simulation structure and dimensions are given in Fig. 9.

together. The results herein not only provide insight into the characteristics of the SPL mode on a structured perfectly conducting surface, but also demonstrate a new and valuable approach to designing electromagnetic metamaterials with particular SPL mode characteristics.

\section{Conclusions}

In this paper, the SPL modes on a structured perfectly conducting surface are reported and investigated. Such SPL modes are electromagnetic surface eigenmodes supported by the structured conductors and can be excited by an electron beam that provides sufficient in-plane moment and energy, like the SP on a metal surface. The standard eigenvalue-solving method based on the finite-difference grid is adopted to solve the SPL modes. The field patterns of the SPL modes in the square holes for in-plane wavevectors $k_{x}=2 \pi / 2 d$ and $k_{x}=2 \pi / d$ are $\mathrm{TE}_{10}$-like and $\mathrm{TE}_{11}$, respectively. However, due to the field coupling between neighboring holes, the field patterns can no longer be identified as any particular waveguide mode for other in-plane wavevectors. The electromagnetic fields of the SPL modes are maximal at the surface of the structured conductor and decay exponentially into both the air and the conductor. The dispersion relations of the SPL modes asymptotically approach the light line from the right at low frequencies, which is like the SP. But except for $k_{x}=2 \pi / d$, the modes of the second branch are not truly the SPL modes. The dispersion relations of the SPL modes in this work agree with the analytical equation derived by Pendry well in the long-wavelength limit. However, the change in mode character with wavevector prevents the dispersion relation from being derived by assuming only the fundamental mode in the holes. On a thin perfect conductor perforated with structures, the SPL mode splits into a high-frequency antisymmetric mode and a low-frequency symmetric mode, which is caused by the mutual interaction of the electromagnetic evanescent fields on both sides.

\section{Acknowledgments}

This work was supported in part by the National Science Council of the Republic of China under Contract No. NSC95-2112-M-006-015-MY3 and the Ministry of Economic Affairs of the Republic of China under Contract No. 95-EC-17--08-S1-0006. The authors gratefully acknowledge valuable suggestions from Professor Din Ping Tsai at National Taiwan University and Professor Wei-Chih Liu at National Taiwan Normal University. The support from National Center for High-performance Computing for providing computer facilities is also acknowledged. 